\title{
Comparison of Co-Peptin, Heart Fatty Acid Binding Protein, Troponin-I and CK-MB Assays as Diagnostic Biomarkers in Patients with Myocardial Infarction
}

\author{
Dr Anisha Sharma ${ }^{1}$, Dr Binita Goswami ${ }^{2}$, Dr Archana Moni ${ }^{3}$, Dr Medha Rajappa ${ }^{4}$ \\ ${ }^{1}$ Registrar, Department of Biochemistry, Lady Hardinge Medical College, New Delhi, India \\ ${ }^{2}$ Associate Professor, Department of Biochemistry, Lady Hardinge Medical College, New Delhi, India \\ ${ }^{3}$ Registrar, Department of Biochemistry, Jawahar Institute of Postgraduate Medical Education \& Research, Pudducherry \\ ${ }^{4}$ Associate Professor, Department of Biochemistry, Jawahar Institute of Postgraduate Medical Education \& Research, Pudducherry
}

\begin{abstract}
Introduction: Coronary artery disease (CAD) is the biggest non- communicable epidemic facing the world today. Myocardial infarction is a common presentation with increased mortality if the diagnosis and subsequent management is not prompt. There is an urgent need to identify a biomarker or a battery of biomarkers which can help the clinician in early diagnosis with reasonable sensitivity and specificity. This study compared the efiicacy of co-peptin, heart fatty acid binding protein, troponin -I and CK-MB as diagnostic markers for myocardial infarction. Methods: The present study was undertaken with 60 acute myocardial infarction patients 60 age and gender matched healthy subjects in a tertiary care hospital. Co-peptin and Heart Fatty Acid Binding Protein levels were estimated by commercially available ELISA kits provided by DRG International, Germany. Troponin I levels were quantitated on automated electrochemiluminescence provided by Roche, Germany using commercially available kits. CK-MB was quantified on Beckmann Coulter AU400 using commercially available kit. Results: The serum levels of co-peptin, heart fatty acid binding protein, troponin -I and CK$M B$ were significantly higher in the cases as compared to controls. Maximum area under the curve is covered by Co-peptin and Troponin (p<0.01). Conclusions: Our study demonstrated that troponin I was the best marker for MI with the highest area under curve followed by co peptin and then $H F A B P$.
\end{abstract}

Keywords: Myocardial infarction; Troponin I;Co-peptin, Heart fatty acid binding protein; ROC

\section{Introduction}

Coronary artery disease (CAD) is emerging as the new epidemic taking various shapes mostly affecting the Indian population at a very young age. As on date, India is said to be the largest contributor of mortality due to CAD. Over the past few years, the pathogenesis behind the development of atherogenecity and its manifestations have evolved enormously. It is now evident that the atherosclerotic plaque develops due to the interplay of various pathological mechanisms taking place at the molecular level. Whether the presence or absence of a single etiological factor makes the diagnosis easy or if early diagnosis can be made at preclinical level is still not well explained. Some of the modifiable risk factors of CAD include sedentary lifestyle, calorie rich diet, smoking, alcohol consumption. The metabolic disturbances that occur due to these risk factors along with the inflammatory process, genetic factors and various other factors which initiate and aggravate the atherosclerotic process is still poorly understood.(1,2)

Detection of Acute Myocardial Infarction (AMI) at the earliest is a problem because of the late rise in cardiospecific troponins which usually rise within 6-9 hours from the onset of chest pain. The biomarkers in use for diagnosis of MI are troponin T \& I, creatine kinase-MB fraction, myoglobin, etc. The cardiac troponins have become the cardiac markers of choice for patients with (Acute Coronary Syndrome)ACS.(3)

The troponin complex present on the thin filament of striated muscle is made up of 3 subunits: troponin T, which binds troponin complex to tropomyosin; troponin I, which regulates the interaction of actin and myosin by inhibiting actomyosin adenosine triphosphate activity; and troponin C, the calcium binding subunit of the troponin complex. The cardiac specific troponins are detectable in the serum within 4 to 12 hours after the onset of myocardial necrosis, and depending on the duration of ischemia and reperfusion status, the time duration varies.(4)

CK-MB present in the cytosol, facilitates movement of high energy phosphates into and out of mitochondria. Since its level rises up in a very short time and comes back to normal in 2-3 days, it cannot be used for late diagnosis of MI.(5)

All the above said markers have proved to be beneficial in the diagnosis of MI but they suffer from low sensitivity and specificity. Research scientists are now focussing on novel markers that can detect MI with higher precision and accuracy. Two such new compounds are co-peptin and Heart-type Fatty Acid-Binding Protein (H-FABP).

Co-peptin, a 39 amino acid glycopeptide co-synthesised with arginine vasopressin (AVP) is an upcoming neuro-endocrine peptide which is newly introduced. It is released in 


\section{International Journal of Science and Research (IJSR) \\ ISSN (Online): 2319-7064 \\ Index Copernicus Value (2013): 6.14 | Impact Factor (2015): 6.391}

stoichiometric pattern from the hypothalamus upon release of AVP. In acute stress, co-peptin rises and reflects stress level similar to that of AVP which was known for mediation of non-specific stress conditions in addition to its role in water homeostasis. AMI, an acute stress condition shows elevated levels of plasma co-peptin.(5)

Heart-type Fatty Acid-Binding Protein (H-FABP), a small cytoplasmic protein $(15 \mathrm{kDa})$ released from cardiac myocytes following ischaemia is involved in active fatty acid metabolism where it transports fatty acids from the cell membrane to mitochondria for oxidation.(6) It may also be responsible for regulation of cell growth and proliferation. It is also a sensitive biomarker for myocardial infarction (7) and can be detected in the blood within 1-3 hours of onset of chest pain.(8)

Previous reports show that there has been an extensive research going on to discover new biomarkers that will help us to group patients who are more prone to complications of atherogenesis and others who do not develop complications. The atherosclerotic pathway as well as the impending complications have further necessitated the identification of novel biomarkers that could assess the kinetics of atherosclerosis.(9) There is a great demand in identifying such a biomarker in our context taking into account the geographical diversity and the meagre resource available to the population at large. Hence, it is all important to identify a single marker which will help in early diagnosis and prognosis of myocardial infarction which presents as acute episodes as a consequence of CAD.

The present study was conducted to determine the diagnostic efficacy of co-peptin, Heart-type Fatty Acid-Binding Protein and compare them with the conventional markers CK-MB and troponin in subjects with myocardial infarction.

\section{Materials and Methods}

The present study was undertaken in a tertiary health care facility in India after approval from the Institute Ethics Committee. 60 acute myocardial infarction patients attending our hospital and 60 age and gender matched healthy subjects were recruited into the study, after obtaining written informed consent from all study subjects.

The patients with MI were diagnosed according to the established protocol in the cardiac care unit by trained medical fraternity. A detailed history and examination was also carried out by the medical staff in the CCU. Blood samples were withdrawn under aseptic conditions after getting written informed consent. The serum was separated, aliquoted and stored till further analysis. The controls were chosen among the healthy volunteers from the hospital staff who agreed to be a part of the study. Any previous history suggestive of MI, diabetes mellitus and concomitant illness amounted to exclusion from the study. The blood samples obtained were then processed and stored for further analysis.

The parameters analysed in our study included co-peptin, Heart fatty Acid Binding Protein, Troponin-I and CK-MB. Co-peptin and Heart Fatty Acid Binding Protein levels were estimated by commercially available ELISA kits provided by DRG International, Germany. Troponin I levels were quantitated on automated electro-chemiluminescence provided by Roche, Germany using commercially available kits. CK-MB was quantified on Beckmann Coulter AU400 using commercially available kit.

\section{Data Analysis}

Data was expressed as mean \pm SD. Normal data was

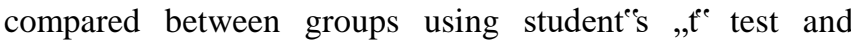
correlation analysis was done to assess the degree of association between the parameters and the disease condition. Statistical analysis was done on SPSS version 17.0. "p" value less than 0.05 was considered statistically significant.

\section{Results}

Table no 1 shows the demographic characteristics of the study population. As depicted in table 2, levels of co-peptin in confirmed cases were raised in comparison to controls taken (Cases-26.2 pmol/l , Controls-6.06 pmol/l), Heart Fatty Acid Binding Protein (Cases-310.2 ng/ml , Controls$35.31 \mathrm{ng} / \mathrm{ml}$ ), Troponin I (Cases-7.31 ng/ml , Controls-0.02 $\mathrm{ng} / \mathrm{ml}$ ) and CK-MB (cases-80.5 ng/ml , Controls-10.6 $\mathrm{ng} / \mathrm{ml})$. This difference was highly significant $(<0.001)$. Table 2 shows the Receiver Operating Characteristics (ROC curve). Maximum area under the curve is covered by Copeptin and Troponin $(\mathrm{p}<0.001)$.

Table 1: Demographic characteristics of the study population

\begin{tabular}{|c|c|c|}
\hline Characteristic & $\begin{array}{c}\text { Controls } \\
(\mathrm{n}=60)\end{array}$ & $\begin{array}{c}\text { Cases } \\
(\mathrm{n}=60)\end{array}$ \\
\hline Age & $47.7 \pm 12.8$ & $45.9 \pm 13.7$ \\
\hline Sex (M/F) & $50 / 10$ & $52 / 08$ \\
\hline Smoking (Y/N) & Apr-56 & $41 / 19$ \\
\hline $\begin{array}{c}\text { Alcohol consumption (Y/N) } \\
\text { Hypertension/ intake of anti hypertensive } \\
\text { drugs(Y/N) }\end{array}$ & Aug-52 & $22 / 38$ \\
\hline $\begin{array}{c}\text { Dyslipidemia/ intake of lipid lowering } \\
\text { drugs(Y/N) }\end{array}$ & Jan-59 & $25 / 35$ \\
\hline Diabetes Mellitus(Y/N) & $0 / 60$ & Dec-48 \\
\hline Past h/o any cardiovascular event(Y/N) & $0 / 60$ & Aug-52 \\
\hline Family h/o cardiovascular diseases(Y/N) & $0 / 60$ & May-55 \\
\hline
\end{tabular}

Table 2.Serum levels of parameters under study in cases and control

\begin{tabular}{|c|c|c|c|}
\hline Marker & $\begin{array}{c}\text { Controls } \\
(n=60)\end{array}$ & $\begin{array}{c}\text { Cases } \\
(n=60)\end{array}$ & p value \\
\hline Co-peptin (pmol/l) & $6.06 \pm 1.33$ & $26.2 \pm 5.01$ & $<0.001$ \\
\hline $\begin{array}{c}\text { Heart fatty acid binding protein } \\
\text { (ng/ml) }\end{array}$ & $35.31 \pm 6.99$ & $310.2 \pm 91.3$ & $<0.001$ \\
\hline Troponin-I (ng/ml) & $0.02 \pm 0.01$ & $7.31 \pm 2.37$ & $<0.001$ \\
\hline CK-MB (ng/ml) & $10.6 \pm 3.4$ & $80.5 \pm 14.2$ & $<0.001$ \\
\hline
\end{tabular}

Table 3: Receiver operating characteristics (ROC) curve for the parameters under study

\begin{tabular}{|c|c|c|}
\hline Marker & $\begin{array}{c}\text { Area under } \\
\text { curve }\end{array}$ & P value \\
\hline Co-peptin (pmol/l) & 0.842 & $<0.001$ \\
\hline Heart fatty acid binding protein $(\mathrm{ng} / \mathrm{ml})$ & 0.814 & 0.01 \\
\hline Troponin-I (ng/ml) & 0.856 & $<0.001$ \\
\hline CK-MB (ng/ml) & 0.728 & 0.01 \\
\hline
\end{tabular}




\section{International Journal of Science and Research (IJSR) \\ ISSN (Online): 2319-7064 \\ Index Copernicus Value (2013): 6.14 | Impact Factor (2015): 6.391}

\section{Discussion}

CAD is the main cause of mortality and disability in this innovative world. Early and right diagnosis is of great importance to enable immediate and intensive treatment which consequently reduces mortality. If treatment is given within 1 hour of onset of symptoms, mortality can be reduced from $9 \%$ to $3 \%$, but if delayed for 3-4 hours, mortality can become five times higher. However, the golden moment for optimal outcome of coronary revascularisation is within 4 hours after the onset of occlusive coronary thrombosis. So early diagnosis is important in patients presenting with acute chest pain. Unfortunately, at least one-fifth of CAD is clinically unrecognized because of the absence of chest discomfort or atypical ECG changes. Early diagnosis of CAD has great significance because the duration of symptoms has a very strong impact on the hospital management strategy. Our goal will require altering the current methods of cardiac biomarkers for identifying such patients.

It is now well known that AMI activates the main hypothalamic stress hormone, arginine vasopressin (AVP) $(2,10)$. However AVP cannot be used as a biomarker due to its unstable nature and short half life. (5). Co-peptin is stored along with AVP in the neurohypophysial vesicles. Hence it is released simultaneously along with AVP. (2). Co-peptin is stable and easier to measure hence co-peptin assay may be considered to be an indicator of AVP levels and the underlying endogenous stress. It was demonstrated by Sayed et al that co-peptin levels above the diagnostic cut off level of $<14 \mathrm{pmol} / \mathrm{L}$ were associated with elevated intermediate term mortality. Hence co-peptin levels help in prognostication of AMI patients. (11) It can also be stated that co-peptin can be regarded as an earlier biomarker of AMI before troponin levels are raised. (12).

Studies have been conducted to evaluate the diagnostic efficacy of co-peptin either alone or in combination with the established bio-markers of AMI. Nursalim et al demonstrated a negative predictive value of up to $99.7 \%$ using the combination of low level of co-peptin and low level of hs-cTnT for ruling out AMI. BIC-8 is the first interventional clinical trial that studied the rationale of discharging suspected ACS patients who test negative for troponin and co-peptin. Positive correlation between TnT and co-peptin has been established by many researchers.(12) The CHOPIN trail also demonstrated that co-peptin helps in the early diagnosis of myocardial infarction. Giavarina et al concluded the superiority of co-peptin over myoglobin for the early diagnosis of MI from their study on 80 subjects who presented to the emergency department.(13)

HFABP is a small cytosolic protein found in the cardiac muscle which is released into the blood stream in the eventuality of cardiac ischemia as observed in MI. Kaulgud et al reviewed biomarkers for acute coronary syndromes and concluded that elevated HFABP can act as an indicator of adverse cardiac event and is more valuable than myoglobin levels.(14) Moreover, HFABP is more specific for cardiac etiology as compared to myoglobin due to its relative abundance in cardiac muscle. Reiter et al conducted a study on 1247 consecutive patients with suspected AMI who presented to the emergency department. They assessed the diagnostic and prognostic performances of hFABP, copeptin and hs-cTnT in these patients. They concluded that HFABP and co-peptin do not improve the diagnosis of patients with chest pain without ST-segment elevation. Nonetheless they were good biomarkers for risk stratification.(15)

Our study demonstrated that troponin I was the best marker for MI with the highest area under curve followed by co peptin and then HFABP. The major drawback of our study is that we did not follow the patients; hence the prognostic abilities of these markers could not be assessed.

\section{References}

[1] Mallika V, Goswami B, Rajappa M. Atherosclerosispathophysiology and role of novel risk factors: a clinico-biochemical perspective. Angiology. 2007;58:513-522.

[2] Wood D. Established and emerging cardiovascular risk factors. Am Heart J. 2001;141:S49-57.

[3] Elshafei A, Abdalla G, El-Motaal OA ,Salman T. Copeptin: A Neuroendocrine Biomarker in Acute Myocardial Infarction Ann Rev \& Res Biol 2013; 3(4):2231-4776.

[4] Mirzaii-Dizgah I, Riahi E. Salivary troponin I as an indicator of myocardial infarction. Indian J Med Res 2013;138: 2013; 861-865

[5] Ni N, Ranlov P,Weis-Fogh J. Evaluation of four different serum enzymes in the diagnosis of acute myocardial infarction.Br Heart J:1965:27;520-6.

[6] Kleine AH, Glatz JF, Van Nieuwenhoven FA, Van der Vusse GJ . Release of heart fatty acid-binding protein into plasma after acute myocardial infarction in man .Mol Cel Biochem:1992;155-62.

[7] Tanaka T, Hirota Y,Sohmiya K,Nishimura S,Kawamura K. Serum and urinary human heart fatty acid binding protein in acute myocardial infarction. Clin Biochem :1991:24(2):195-201.

[8] Bruins Slot MH, Reitsma JB, Rutten FH, Hoes AW, van der Heijden GJ. Heart-type fatty acid-binding protein in the early diagnosis of acute myocardial infarction: a systematic review and meta-analysis. Heart. 2010 ;96(24):1957-63.

[9] Blake GJ, Ridker PM. C-reactive protein and other inflammatory risk markers in acute coronary syndromes. J Am CollCardiol. 2003;41:37-42.

[10] Kageyama K, Suda T. Regulatory mechanisms underlying corticotropin-releasing factor gene expression in the hypothalamus. Endocr J. 2009;56(3):335-44.

[11]El Sayed ZH, Mahmoud HA, El Shall LY ,El Sheshtawey FA, Mohamed MA. Impact of copeptin on diagnosis of acute coronary syndrome. Egyptian J Med Hum Genet. 2014;15(3), 241-247.

[12] Giavarina D, Carta M, Fortunato A, Wratten ML, Hartmann O, Soffiati G. Copeptin and high sensitive troponin for a rapid rule out of acute myocardial infarction? Clin Lab. 2011;57(9-10):725-30.

[13] Maisel A, Mueller C, Neath SX, Christenson RH, Morgenthaler NG, McCord J,et al. Copeptin helps in the early detection of patients with acute myocardial infarction: primary results of the CHOPIN trial 


\section{International Journal of Science and Research (IJSR) \\ ISSN (Online): 2319-7064}

Index Copernicus Value (2013): 6.14 | Impact Factor (2015): 6.391

(Copeptin Helps in the early detection Of Patients with acute myocardial INfarction). J Am Coll Cardiol. 2013 Jul 9;62(2):150-60

[14] Kaulgud RS, Vijayalxmi P B, Arun B S, Supriya Rao R and Vigneshwar M. Novel biomarkers for risk stratification of acute coronary syndromes. International Journal of Biomedical Research 2015; 6(08): 539-545.

[15] Reiter M, Twerenbold R, Reichlin T, et al. Heart-type fatty acid-binding protein in the early diagnosis of acute myocardial infarction. Heart 2013; 99: 708-714. 